\title{
Modelo de Gestión Innovadora en un Centro Tecnológico Mexicano de Investigación y Desarrollo
}

\author{
Alímagdiel S. Colunga*(1), Víctor M. Molina ${ }^{(2)}$ y Eyrán R. Díaz ${ }^{(2)}$ \\ (1) Corporación Mexicana de Investigación en Materiales. Ciencia y Tecnología 790, Col. Saltillo 400. \\ CP 25290. Saltillo, Coahuila, México. \\ (2) Facultad de Contaduría y Administración, Depto. de Posgrados de Calidad. Universidad Autónoma de \\ Coahuila, Unidad Torreón. Blvd. Revolución 151 Ote. CP 27250. Torreón, Coahuila, México.
}

${ }^{*}$ Autor a quien debe ser dirigida la correspondencia

Recibido Sep. 5, 2017; Aceptado Nov. 27, 2017; Versión final Ene. 12, 2018, Publicado Jun. 2018

\begin{abstract}
Resumen
El objetivo del trabajo es la determinación y validación del modelo de gestión innovadora de un Centro Tecnológico de Investigación y Desarrollo que desde hace 20 años ha demostrado ser auto-sostenible financieramente. Es una investigación no experimental de tipo transversal, exploratoria, y correlacional que determinó la validez convergente y discriminante y la confiabilidad interna del modelo, empleando el modelado de ecuaciones estructurales mediante mínimos cuadrados parciales (MCP). Las cuatro hipótesis formuladas alcanzaron valores de $t$ estadísticos superiores a 1.96, todas las cargas factoriales fueron superiores a 0.7. A partir de estos resultados del estudio, se concluyó que este centro logró asociar sus procesos de gestión tecnológica con el enfoque al cliente basado en sus demandas y expectativas, con su proceso de negocio y con una administración eficaz por proyectos en una operación financieramente autosostenible.
\end{abstract}

Palabras clave: gestión innovadora; centro tecnológico de investigación; auto-sostenible financieramente; mínimos cuadrados parciales; enfoque al cliente

\section{Innovative Management Model in a Mexican Technological Research and Development Center}

\begin{abstract}
The objective of the work is the determination and validation of an innovative management model of a Mexican Technological Research and Development Center that for 20 years has proved to be self-sustaining financially. It is a cross-sectional, exploratory, and correlational non-experimental investigation that determined the convergent and discriminant validity and the internal reliability of the model, employing the modeling of structural equations using partial least squares (PLS). The four hypotheses formulated reached "t" statistic values higher than 1.96 and all factorial loads were higher than 0.7. Based on these results, it was concluded that this center was able to associate its technological management processes with the customer focus based on its demands and expectations, with its business process and with its effective management by projects in a financially self-sustainable operation.
\end{abstract}

Keywords: innovative management; technological research center; financially self-sustainable; partial least squares; customer focus 


\section{INTRODUCCION}

La auto-suficiencia financiera con utilidades por encima del promedio de la industria de que se trate, ha sido la preocupación permanente de las empresas de base tecnológica, diferenciándose de la competencia a través del desarrollo de tecnología propia, innovaciones y/o productos que reemplacen a los actuales, buscando satisfacer las necesidades más apremiantes de sus clientes e, inclusive, creando demandas mediante la interacción intensiva, con el propósito de demostrar la ventaja de sus productos y servicios ante la competencia. Asimismo, un Centro Tecnológico de Investigación y Desarrollo (CTID) no es ajeno a las presiones externas del mercado y competidores, siendo fundamental para su supervivencia lograr la autosostenibilidad financiera generando más conocimientos y aplicándolos a la mayor velocidad posible. Por esta razón, dirigen sus esfuerzos a desarrollar tecnologías y metodologías innovadoras con el objeto de diferenciarse de la competencia y procurar así condiciones de comercialización más ventajosas.

En México los CTID tienen como finalidad desarrollar competencias científicas y tecnológicas y fueron creados para detonar el desarrollo local y regional de la industria, afirman Estrada y Aguirre (2013). Sin embargo, el marco legal es complejo y limitativo por lo que resulta complicado realizar alianzas que coadyuven y amplíen su oportunidad de desarrollo en el entorno nacional e internacional, así como seleccionar y atender a clientes potenciales. Las principales actividades que realizan los CTID nacionales se pueden sintetizar en cuatro, según Nath y Mrinalini (2002) y Merritt (2007): 1) Servicios de investigación y desarrollo experimental. 2) Trabajos de consultoría y generación de información técnica. 3) Servicios de calibración, medición y análisis. 4) Entrenamiento y capacitación de personal técnico industrial. Al respecto, Merritt (2007) sostiene que estos servicios se ven afectados por el grado de desarrollo de las empresas medianas de la industria regional, quienes son sus principales clientes, por lo que se limita a consultoría y servicios especializados, como calibración, medición y análisis, a empresas industriales rezagadas tecnológicamente, donde la relación comercial y científica adquiere un menor alcance.

No obstante, se perciben áreas de oportunidad en la vinculación con la industria mejor equipada y en el apoyo para detonar el crecimiento en la industria con menos recursos, sin dejar de lado la empresa mediana, con ello se impulsa el círculo virtuoso al lograr que la industria local crezca y en consecuencia aumente la inversión en ciencia y tecnología. Para alcanzar este círculo virtuoso se tiene que determinar metodológicamente cuáles son las fortalezas que un CTID tendrá que crear o desarrollar, tomando en cuenta que el marco legal es una limitante externa, por ser dependencias del gobierno federal. Las restricciones a la búsqueda de crecimiento y/o auto-sostenibilidad financiera deben superarse realizando revisiones y adecuaciones necesarias en las reformas del marco legal.

Ahora bien, las capacidades y las competencias son componentes necesarios e importantes en la estructuración de estrategias de desarrollo tecnológico, refieren Castellanos (2007) y Casanueva (2001). Según Hlupic (2002), el conocimiento en los niveles de gestión táctico y estratégico de la organización se manifiesta en la forma de capacidades y competencias. De acuerdo con Hlupic (2002), se definen las competencias como las habilidades organizacionales y técnicas involucradas en la obtención de cierto nivel de rendimiento en la producción mientras que las capacidades son las que describen las habilidades estratégicas en la aplicación e integración de competencias.

El problema identificado en la presente investigación es la falta de definición de un modelo de gestión para los CTID mexicanos que les permita operar sin recursos del presupuesto gubernamental, enfocándose en las necesidades del mercado, a fin de mejorar el impacto (vinculación) en la industria.

Este problema no es un hecho aislado ya que sólo el Consejo Estatal de Ciencia y Técnica (CONACYT) de México tiene 16 centros de investigación que trabajan con recursos federales, sin contar decenas de otros Centros de Investigación y Desarrollo pertenecientes a distintas Secretarías de Estado que presentan una situación similar. Por consiguiente, la implantación de un modelo de gestión innovadora en Centros Tecnológicos de Investigación y Desarrollo Auto-sostenibles financieramente (CTID-AS), sobre todo en el caso de los que no tienen apoyos presupuestales de la federación o aspiran a no tener estos apoyos para su funcionamiento, presenta una oportunidad de estudio y de generación de conocimiento, razón por la cual se desarrolló un proyecto de investigación, que en su primera parte tuvo como objetivos identificar y validar las capacidades distintivas para el éxito y competitividad de estos centros.

La base de datos empleada para la obtención del actual modelo de gestión innovadora es la misma utilizada para la determinación de las capacidades distintivas de los CTID referida en Colunga (2016), donde se comprobó la existencia de cuatro factores determinantes para el éxito y competitividad que se identificaron como: Gestión Tecnológica; Enfoque al Cliente; Procesos Clave de Negocios y Administración de Proyectos, asociados a 18 variables que identifican los factores clave de éxito. 
La Tabla 1 es una traducción y adecuación de los resultados previamente comprobados en Colunga (2016) y expone los factores y variables que se emplean en este artículo. Esta base de datos fue obtenida de las respuestas de 55 de los 70 jueces que fueron convocados, considerando como tales a expertos con grado de doctorado y maestría, que trabajan para 22 instituciones de reconocida producción científica, educativa y tecnológica diferentes; estos 55 expertos (7 con Postdoctorado, 28 con Doctorado, 13 con Maestría y 7 con Licenciatura) conformaron el $80 \%$ de la población en capacidad de responder este instrumento dada su experticia en estos temas. Este ejercicio se realizó en el I trimestre del año 2016.

Tabla 1: Factores y variables identificados en los CTID.

\begin{tabular}{|c|c|c|}
\hline Factores determinantes & Variables & Identificador de la variable \\
\hline \multirow{5}{*}{$\begin{array}{c}\mathrm{F}_{1} \\
\text { Gestión tecnológica }\end{array}$} & $\mathrm{V}_{1}$ & Planeación tecnológica \\
\hline & $\mathrm{V}_{2}$ & Criterios explícitos de asignación de prioridades y recursos \\
\hline & $V_{3}$ & Implantación \\
\hline & $\mathrm{V}_{4}$ & Adquisición, asimilación y desarrollo de tecnología \\
\hline & $\mathrm{V}_{5}$ & Protección del patrimonio de la organización \\
\hline \multirow{3}{*}{$\begin{array}{c}\mathrm{F}_{2} \\
\text { Enfoque al cliente }\end{array}$} & $\mathrm{V}_{10}$ & Relaciones con clientes \\
\hline & $\mathrm{V}_{11}$ & Portafolio de servicios \\
\hline & $V_{12}$ & Propuesta de valor \\
\hline \multirow{4}{*}{$\begin{array}{c}\mathrm{F}_{3} \\
\text { Procesos clave } \\
\text { de negocios }\end{array}$} & $V_{6}$ & Cultura colaborativa \\
\hline & $\mathrm{V}_{7}$ & Comercialización de tecnología \\
\hline & $\mathrm{V}_{8}$ & Licenciamiento de tecnología propia \\
\hline & $\mathrm{V}_{9}$ & Creación de empresas de base tecnológica (spin off) \\
\hline \multirow{6}{*}{$\begin{array}{c}\mathrm{F}_{4} \\
\text { Administración de } \\
\text { proyectos }\end{array}$} & $V_{13}$ & Sistema de administración de proyectos \\
\hline & $\mathrm{V}_{14}$ & Recursos humanos altamente calificados \\
\hline & $V_{15}$ & Infraestructura de laboratorios de i + D \\
\hline & $\mathrm{V}_{16}$ & Inteligencia tecnológica \\
\hline & $V_{17}$ & Administración de riesgos \\
\hline & $V_{18}$ & Sistema de evaluación del desempeño \\
\hline
\end{tabular}

Los sustentos teóricos de los factores determinantes asociados al modelo de gestión innovadora son: las definiciones de la gestión tecnológica referidas por Aguilar y Yepes (2006), García et al. (2008), Intxaurburu y Velasco (2010), Leitner y Warden (2004) y Castellanos (2007); el enfoque al cliente planteado por Cabanelas et al. (2007), Gil-Lafuente y Luis-Bassa (2011) y Castillo (2009); la administración de proyectos conceptualizada en Sánchez y Álvarez (2005) y Braun et al. (2000). Álvarez (2003), Barragán (2010) y Rigby (2015) reseñan los procesos clave de negocios.

Todos los factores del modelo de gestión innovadora forman parte de un sistema en el cual la intención de auto-sostenibilidad financiera está latente. El modelo parte de la identificación de las fortalezas de este Centro con énfasis en la "gestión tecnológica" $\left(F_{1}\right)$ que prioriza el sector de ingeniería a través de investigación y desarrollo tecnológico, estudios y proyectos de ingeniería y, capacitación y estudios de posgrado. Se "enfoca en clientes" $\left(F_{2}\right)$ tales como empresas, cámaras y sectores del gobierno afines al mismo campo de operación, que disponen tanto de infraestructura pública como industrial. Realiza de manera eficaz "procesos clave de negocios" $\left(F_{3}\right)$ en donde la competencia clave "cultura colaborativa", por mencionar un ejemplo, gana en calidad cada vez más a través de nuevas herramientas de tecnologías de información, y ellos se asocian a la "administración de proyectos" $\left(\mathrm{F}_{4}\right)$ de manera innovadora pues se requiere una administración de los recursos de la empresa u organización a través de sistemas de información automatizados y en línea, que están en constante evolución innovadora.

A partir de la revisión teórica, la comprensión del modelo de gestión innovadora y los estudios empíricos, para la investigación se formulan las hipótesis siguientes: $\mathrm{H}_{1}$ : En el modelo conceptual de gestión de un CTID-AS la adecuada gestión tecnológica tiene una influencia positiva en el enfoque al cliente. $\mathrm{H}_{2}$ : En el modelo conceptual de gestión de un CTID-AS el adecuado enfoque al cliente basado en sus demandas y expectativas, tiene una influencia positiva en la administración del proyecto que se ejecutará. $\mathrm{H}_{3}$ : En el modelo conceptual de gestión de un CTID-AS el adecuado enfoque al cliente tiene una influencia positiva en 
los procesos de negocios, es decir, en la generación de valor económico a través de dichos procesos de negocio, con base en la satisfacción de las necesidades del cliente. $\mathrm{H}_{4}$ : En el modelo conceptual de gestión de un CTID-AS el adecuado proceso de negocio tiene una influencia positiva en la administración de proyecto.

\section{MATERIALES Y MÉTODOS}

Derivado de los resultados obtenidos en Colunga (2016), en la Fig. 1 se muestra el modelo conceptual que se propone y las hipótesis a comprobar. La investigación contempló la combinación de métodos cualitativos y cuantitativos. Es un estudio correlacional donde se contrastaron las cuatro hipótesis de investigación aplicando un análisis predictivo y Regresión Múltiple por Mínimos Cuadrados Parciales (MCP, o PLS por sus siglas en inglés) lo que permitió sustentar y explicar el modelo de gestión innovadora para un CTID financieramente auto-sostenible (CTID-AS).

El uso de MCP en la validación del modelo está sustentado en el carácter predictivo de la investigación. "Cepeda y Roldán (2005); apuntan que MCP, a diferencia del modelo de covarianzas (MBC), se orienta principalmente al análisis causal-predictivo en situaciones de alta complejidad, pero baja información teórica" y mencionan otras razones tales como: variables con distribuciones no normales, investigación no experimental con datos obtenidos de encuestas, muestra de estudio no muy amplia y una teoría que aún no se encuentra desarrollada de una manera sólida.

Los autores Roldán y Sánchez-Franco (2012) crean una discusión acerca de las diferencias clave entre los Modelos de Ecuaciones Estructurales (SEM), basados en covarianza y los que son basados en varianza como MCP. También Nijssen y Douglas (2011), Rodríguez-Pinto et al. (2008) reafirman la posibilidad de emplear PLS con pequeñas muestras y frecuencias de distribución no necesariamente normales. Otros autores, Chin (1998) y Gónzalez et al. (2006) sugieren emplear MCP en lugar de AMOS (de SPSS) o EQS en muestras relativamente pequeñas; (Aldás-Manzano, 2012; Chin et al., 2003 y Hair et al., 2011) afirman que el MCP se recomienda generalmente en modelos de investigación cuando el objetivo es la predicción en lugar de la confirmación de las relaciones estructurales, con un conocimiento teórico poco desarrollado por ser una teoría naciente y todos los indicadores correlacionados.

A pesar de la crítica reciente hacia el método, Rönkkö (2014) menciona que, la mayoría de los estudios existentes que analizan el funcionamiento de MCP han alcanzado conclusiones positivas. Mclntosh (2014), concluye que los modelos MCP son viables con enfoques de Modelos de Ecuaciones Estructurales (SEM) para estimar y evaluar el modelo teórico. Henseler (2016) señala que el modelado de MCP es una técnica de modelado de ecuaciones estructurales basada en covarianza (SEM) que se aplica ampliamente en las ciencias empresariales y sociales.

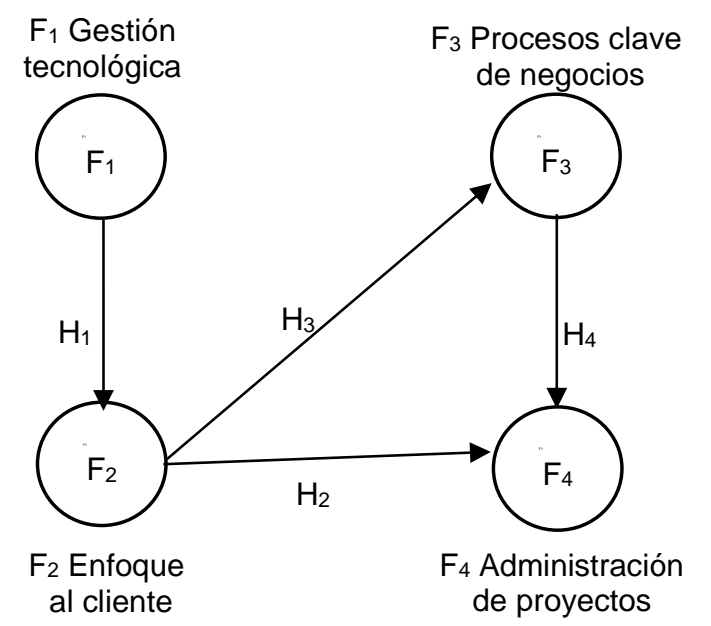

Fig. 1: Modelo estructural de la gestión innovadora de un CTID. Modelo conceptual basado en un formato de Kwong (2013).

Descripción del procedimiento de MCP.

El procedimiento que se explica a continuación fue organizado -en general- cumpliendo los requerimientos del Software SmartPLS (V.3.2.7) de Ringle (2015). Este proceder metodológico se muestra en la Fig. 2. Los autores de este artículo enriquecieron el procedimiento empleando soluciones de Cepeda y Roldán (2005) y Miranda-Zapata y Ruíz (2015). Un elemento que coadyuvó a la comprensión del mismo fue también el 
conocimiento previo que los autores poseen de los modelos de estructuras de covarianzas, (Molina, 2017) y los enfoques de Aldás-Manzano (2012), lo que facilitó asimilar de manera más rápida los MCP que tienen como objetivo la predicción de las variables latentes no basadas en la covarianza, sino que realizan estimaciones de mínimos cuadrados ordinarios a partir de los análisis de componentes principales (ACP) como se trabajó previamente en Colunga (2016).

En esta investigación se consideró que el modelo a emplear pertenece a un constructo reflectivo, dado que cumplió una recomendación de Chin (1998), cuando orienta que, si se incrementa uno de los indicadores en una dirección, el resto de los indicadores también cambia de forma similar. Además, se comprobó que las medidas de un constructo están correlacionadas y alcanzaron un alto nivel en medidas de consistencia interna. Previo a la aplicación del procedimiento MCP se debe comprobar que los datos no sigan una distribución normal, empleando el estadístico Kolmogorov - Smirnov (K-S de una muestra). La regla indica que cuando valores K-S son de 0.00 , la distribución no mantiene un comportamiento normal, cayendo entonces dentro del campo de la estadística no paramétrica.
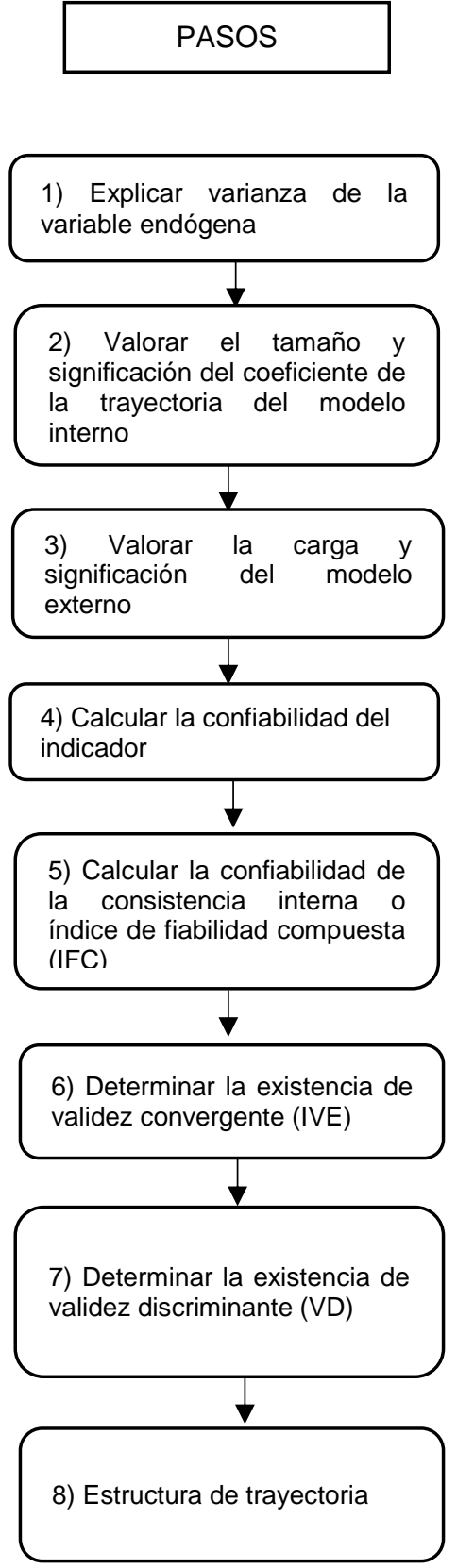

Las variables endógenas son aquellas a partir de las cuales parten las trayectorias, en este caso se consideró la variable Gestión tecnológica, $F_{1}$ cuyo valor de varianza se obtiene de la desviación estándar expresada como STDEV²

Los coeficientes de trayectoria del modelo interno, se consideran significativos si " $t$ " estadístico es mayor que 1.96 con $5 \%$ como nivel de significancia.

Hair (2011) recomienda que la carga factorial sea superior a 0.7 (a diferencia de EQS que admite valores por encima de 0.6 ) y obtener un valor " $\mathrm{t}$ " estadístico mayor que $1.96 \mathrm{con}$ $5 \%$ como nivel de significancia.

Hair (2011) recomienda que el nivel mínimo aceptable de la confiabilidad del indicador sea de 0.4 y superior a 0.7 el nivel preferido.

Como Alpha de Cronbach, para cada factor separado, no tiene en cuenta la influencia sobre la fiabilidad del resto del constructo). Fornell y Larcker (1981) recomiendan el (IFC) como la varianza extraída entre el grupo de variables observadas y el constructo fundamental. Bagozzi y Yi (1988) recomiendan un IFC superior a 0.60.

La validez convergente se mide por el índice de la varianza media extraída (IVE) que debe ser superior a 0.50 y se obtiene como salida de la corrida del Algoritmo MCP.

Una escala tiene VD si los instrumentos diseñados para medir un constructo dado no sirven para medir otros constructos Bentler (1980) indica que es comprobada cuando las cargas de los indicadores son superiores a todas las cargas cruzadas de otros constructos (Hair, 2013). Fornell y Larcker (1981) comprueban VD, si $\left(\right.$ IVE $^{1 / 2}$ ) en cada variable latente es mayor a la correlación entre las variables latentes.

Según Kwong (2013), ésta es confiable si supera un valor de 0.4 y "p" es significativo para valores inferiores a 0.05 . Aplicar "re-muestreo" ("bootstrapping").

Fig. 2 Procedimientos para la ejecución de la estimación del modelado de trayectorias (paths) 


\section{Procedimientos complementarios a considerar en la metodología}

El poder predictivo de un modelo se estimó para las variables latentes dependientes mediante el estadígrafo $\mathrm{R}^{2}$, que ha de ser interpretado de la misma forma que los obtenidos en un análisis de regresión múltiple, este coeficiente calcula una media de relación lineal entre dos variables aleatorias, que puede tomar valores entre -1 y +1 ; un valor de 0 significa no correlación y por convención, los valores que están por encima de 0.7 se consideran óptimos. Falk y Miller (1992) señalan que valores de $\mathrm{R}^{2}$ menores de 0.1 , aun siendo estadísticamente significativos, proporcionan muy poca información, por lo que las relaciones que se formulan como hipótesis con relación a esta variable latente tienen un bajo nivel predictivo.

Para manejar la multicolinealidad, Chun y Keles (2010) recomiendan MCP, por otra parte, y para comprobar la no existencia de colinealidad, o sea, que ninguna de las variables independientes sea combinación lineal de otras (coeficiente de correlación entre ambas variables $=1$ ), se calcula el factor de inflación de la varianza (VIF). Mandeville (2008) señala que valores de VIF $=1$, indican que no hay colinealidad; $4<\mathrm{VIF}<$ 10 indican colinealidad y VIF > 10, indican colinealidad grave.

\section{RESULTADOS Y DISCUSION}

Por la naturaleza de los resultados se presentan simultáneamente los resultados y la discusión, en el mismo orden de los procedimientos recomendados por Kwong (2013) y que fueron explicados en la metodología. En la primera parte se presentan y discuten los resultados para el modelo interno, Fig. 1 y posteriormente se presenta el análisis del modelo de medida o modelo externo que se muestra en la Fig. 3.

\section{Modelo interno}

Como las variables endógenas son aquellas a partir de las cuales parten las trayectorias, en este caso se considera como tal la variable Gestión tecnológica $\left(F_{1}\right)$ cuyo valor de varianza se obtiene de la desviación estándar expresada como STDEV², alcanzando un valor mínimo de 0.00384. En la Tabla 2, se muestra los datos de salida que entrega la corrida del programa MCP-Inteligente (SmartPLS) en la que se aprecia que los valores del coeficiente de trayectoria superan el valor mínimo de 0.4 ; el valor "p" es inferior a 0.01 para todas las trayectorias.

Tabla 2: Tamaño y significación de la trayectoria del modelo interno.

\begin{tabular}{|c|c|c|c|c|}
\hline Trayectoria & $\begin{array}{l}\text { Coeficiente de } \\
\text { trayectoria }\end{array}$ & $\begin{array}{l}\text { Media de la } \\
\text { muestra (M) }\end{array}$ & $\begin{array}{c}\text { Desviación estándar } \\
\left(\text { STDEV }^{2}\right)\end{array}$ & "p" valores \\
\hline $\begin{array}{l}F_{1} \text { Gestión tecnológica -> } \\
F_{2} \text { Enfoque al cliente }\end{array}$ & 0.818 & 0.812 & 0.075 & 0.000 \\
\hline $\begin{array}{l}F_{2} \text { Enfoque al cliente }-> \\
F_{3} \text { Procesos clave de negocios }\end{array}$ & 0.646 & 0.623 & 0.150 & 0.000 \\
\hline $\begin{array}{l}F_{2} \text { Enfoque al Cliente -> } \\
F_{4} \text { Administración de proyectos }\end{array}$ & 0.502 & 0.499 & 0.125 & 0.000 \\
\hline $\begin{array}{l}\mathrm{F}_{3} \text { Procesos clave de negocios -> } \\
\mathrm{F}_{4} \text { Administración de proyectos }\end{array}$ & 0.414 & 0.395 & 0.144 & 0.004 \\
\hline
\end{tabular}

También el valor de la confiabilidad del indicador (cargas $\left.{ }^{2}\right)$ supera 0.4 como valor recomendado. El Índice de Fiabilidad Compuesta (IFC), como ya se señaló, representa la varianza extraída entre el grupo de variables observadas y el constructo fundamental. Este resultado lo entrega como salida el MCP y alcanza valores: $F_{1}=0.902 ; F_{2}=0.910 ; F_{3}=0.906$ y $F_{4}=0.935$ que son superiores al mínimo recomendado de 0.6.

El valor del índice de la varianza extraída (IVE) representa el valor de la validez convergente y se obtiene como salida de la corrida del Algoritmo MCP. Los valores del IVE son superiores a 0.5 , los de " $\mathrm{t}$ " estadístico son superiores a 3.291 y los de " $p$ " son inferiores a 0.001 . Alfa de Cronbach para todos los factores supera el valor de 0.76 , tal como muestra la Tabla 3 , por lo que se cumple la validez convergente.

Tabla 3: Validez convergente.

\begin{tabular}{|l|c|c|c|c|c|}
\hline \multicolumn{1}{|c|}{ Factores determinantes } & $\begin{array}{c}\text { Índice varianza } \\
\text { extraída (IVE) }\end{array}$ & $\begin{array}{c}\text { Media de la } \\
\text { muestra (M) }\end{array}$ & $\begin{array}{c}\text { Alfa de } \\
\text { Cronbach }\end{array}$ & "t” estadístico & "P” valores \\
\hline$F_{1}$ Gestión tecnológica & 0.669 & 0.652 & 0.876 & 7.277 & 0.000 \\
\hline$F_{2}$ Enfoque al cliente & 0.754 & 0.738 & 0.837 & 8.854 & 0.000 \\
\hline$F_{3}$ Procesos clave de negocios & 0.708 & 0.688 & 0.767 & 7.143 & 0.000 \\
\hline$F_{4}$ Administración de proyectos & 0.706 & 0.655 & 0.876 & 6.492 & 0.000 \\
\hline
\end{tabular}


Para el cálculo de la validez discriminante Tablas 4 y 5 se comprobaron dos criterios, el primero mediante la relación cargas del indicador - cargas cruzadas, en donde el valor de las cargas cruzadas se obtiene como salida al concluir el cálculo de "re-muestreo", luego se accede a Informe MCP, se continúa la ruta hacia validez discriminante y luego a cargas cruzadas. Una muestra parcial de este resultado se presenta en la Tabla 4 donde se comprueba que el valor de las cargas del indicador (columna extrema derecha) es superior al valor de las cargas cruzadas para cada variable observable de su propia fila. Otro criterio es el de Fornell y Larcker (1981) quienes indican que la raíz cuadrada de IVE (IVE ${ }^{1 / 2}$ ) en cada variable latente puede utilizarse para establecer la validez discriminante, si se cumple que es mayor que otros valores de correlación entre las variables latentes. Este resultado se muestra en la Tabla 5.

Tabla 4: Validez discriminante por cargas cruzadas, muestra representativa con solo cuatro variables.

\begin{tabular}{|c|c|c|c|c|c|}
\hline \multirow{2}{*}{$\begin{array}{c}\text { Variable } \\
\text { observable }\end{array}$} & $\begin{array}{c}|c| \\
\mathrm{F}_{1} \\
\text { Gestión } \\
\text { tecnológica }\end{array}$ & $\begin{array}{c}\text { Enfoque al } \\
\text { cliente }\end{array}$ & $\begin{array}{c}\mathrm{F}_{2} \\
\text { Procesos clave } \\
\text { de negocios }\end{array}$ & $\begin{array}{c}\mathrm{F}_{3} \\
\text { Administración } \\
\text { de proyectos }\end{array}$ & $\begin{array}{c}\text { Cargas del } \\
\text { indicador }\end{array}$ \\
\hline $\begin{array}{c}\mathrm{V}_{1} \\
\text { Planeación tecnológica }\end{array}$ & 0.709 & 0.791 & 0.659 & 0.792 & 0.791 \\
\hline $\begin{array}{c}\mathrm{V}_{2} \\
\begin{array}{c}\text { Criterios explícitos de } \\
\text { asignación de prioridades y } \\
\text { recursos }\end{array}\end{array}$ & 0.713 & 0.805 & 0.484 & 0.647 & 0.805 \\
\hline $\begin{array}{c}\mathrm{V}_{3} \\
\text { Implantación }\end{array}$ & 0.619 & 0.875 & 0.635 & 0.689 & 0.875 \\
\hline $\begin{array}{c}\mathrm{V}_{4} \\
\text { Adquisición, asimilación y } \\
\text { desarrollo de tecnología }\end{array}$ & 0.544 & 0.791 & 0.623 & 0.709 & 0.791 \\
\hline
\end{tabular}

Tabla 5: Comprobación de la validez discriminante.

\begin{tabular}{|c|c|c|c|c|c|c|}
\hline $\begin{array}{c}\text { Factores } \\
\text { determinantes }\end{array}$ & IVE & $\begin{array}{c}\text { Raíz } \\
\text { cuadrada } \\
\text { de IVE } \\
\end{array}$ & $\begin{array}{c}F_{1} \\
\text { Gestión } \\
\text { tecnológica }\end{array}$ & $\begin{array}{c}\mathrm{F}_{2} \\
\text { Enfoque al } \\
\text { cliente }\end{array}$ & $\begin{array}{c}\mathrm{F}_{3} \\
\text { Procesos clave } \\
\text { de negocios }\end{array}$ & $\begin{array}{c}\mathrm{F}_{4} \\
\text { Administración } \\
\text { de proyectos }\end{array}$ \\
\hline $\begin{array}{c}F_{1} \\
\text { Gestión } \\
\text { tecnológica }\end{array}$ & 0.754 & 0.860 & & $>0.819$ & $>0.538$ & $>0.630$ \\
\hline $\begin{array}{c}\mathrm{F}_{2} \\
\text { Enfoque al cliente }\end{array}$ & 0.669 & 0.817 & & & $>0.657$ & $>0.465$ \\
\hline $\begin{array}{c}\mathrm{F}_{3} \\
\text { Procesos clave } \\
\text { de negocios }\end{array}$ & 0.708 & 0.841 & & & & $>0.463$ \\
\hline $\begin{array}{c}\mathrm{F}_{4} \\
\text { Administración de } \\
\text { proyectos }\end{array}$ & 0.706 & 0.840 & & & & \\
\hline
\end{tabular}

Una medida del poder predictivo de un modelo es el valor $\mathrm{R}^{2}$ para las variables latentes dependientes. Este índice es interpretado de la misma forma que los $\mathrm{R}^{2}$ obtenidos en un análisis de regresión múltiple (Cepeda y Roldán, 2005). Este valor para el factor $\mathrm{F}_{4}$ es de 0.691, lo que indica que las variables Gestión, Enfoque y Procesos, interpretan el $69.1 \%$ de la varianza en Administración. De igual forma para la variable latente Procesos el valor de $\mathrm{R}^{2}$ es de 0.445 y para la variable Enfoque de 0.669 . Estos resultados demuestran el poder predictivo que tiene el modelo estructural presentado y comprobado.

\section{Modelo externo}

La Fig. 3 muestra los valores de la carga factorial de cada una de las 18 variables a partir del procedimiento de "re-muestreo", todas las cargas son superiores a 0.7 y el valor de la significación de cada una de ellas es inferior a 0.05 , valor recomendado. 


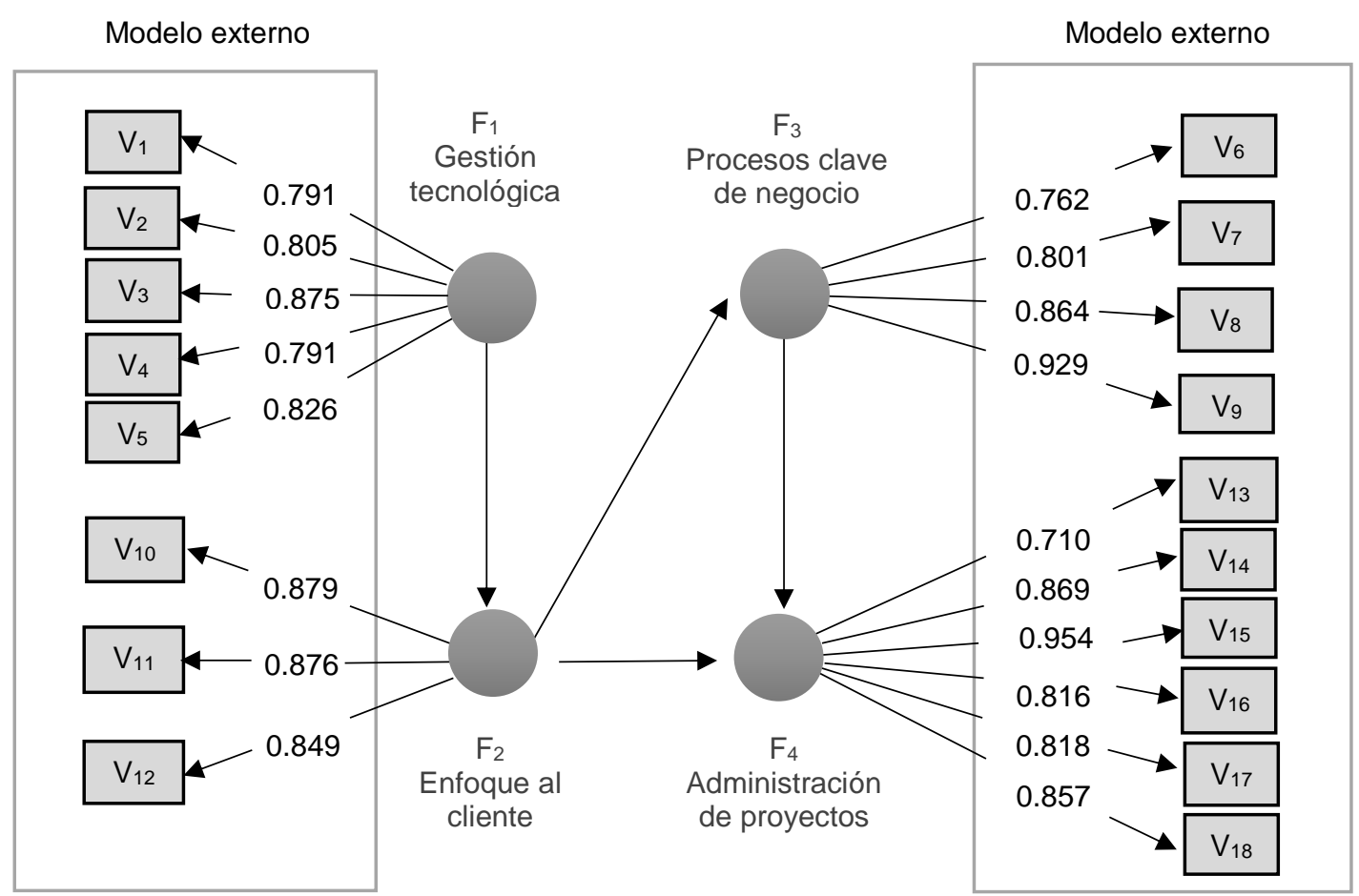

Fig. 3: Carga y significación del modelo externo.

El cálculo del estadístico de colinealidad se halla por medio del procedimiento MCP-Algoritmo, en este caso, ninguna de las cuatro variables del modelo estructural sobrepasa el valor de 1.715 y en el modelo de medida, solo la variable \# 15 alcanza un valor por encima de 4 (7.549), este atípico resultado, aunque debe ser motivo de análisis, no es indicador de colinealidad grave en el modelo.

Una vez comprobada la fiabilidad y validez del modelo de medida y que el coeficiente de trayectoria es superior a 0.4 el proceso de "re-muestreo" valida positivamente las cuatro hipótesis

Tabla 6. Comprobación de hipótesis

\begin{tabular}{|c|l|c|c|}
\hline Hipótesis & \multicolumn{1}{|c|}{ Trayectoria } & \multicolumn{1}{c|}{$\begin{array}{c}\text { Coeficiente de trayectoria } \\
{ }^{*} \mathrm{p}<0.05 ;{ }^{* *} \mathrm{p}<0.01 ;{ }^{* * *} \mathrm{p}<0.001\end{array}$} & "t" valor (re muestreo) \\
\hline $\mathrm{H}_{1}$ & $\begin{array}{l}\mathrm{F}_{1} \text { Gestión tecnológica }-> \\
\mathrm{F}_{2} \text { Enfoque al cliente }\end{array}$ & $0.818^{* * *}$ & 12.727 \\
\hline $\mathrm{H}_{2}$ & $\begin{array}{l}\mathrm{F}_{2} \text { Enfoque al Cliente }-> \\
\mathrm{F}_{4} \text { Administración de proyectos }\end{array}$ & $0.5022^{* * *}$ & 4.008 \\
\hline $\mathrm{H}_{3}$ & $\begin{array}{l}\mathrm{F}_{2} \text { Enfoque al cliente }-> \\
\mathrm{F}_{3} \text { Procesos clave de negocios }\end{array}$ & $0.646^{* * *}$ & 5.022 \\
\hline $\mathrm{H}_{4}$ & $\begin{array}{l}\mathrm{F}_{3} \text { Procesos clave de negocios }-> \\
\mathrm{F}_{4} \text { Administración de proyectos }\end{array}$ & $0.414^{* *}$ & 3.086 \\
\hline
\end{tabular}

La Fig. 3 ofrece los valores "t" de re muestreo para el modelo externo, mostrando que todas las cargas son significativas, lo que refuerza la conclusión de la fiabilidad del modelo de medición y la validez convergente. La Tabla 6 ofrece la estimación del modelo estructural interno con los valores " $\mathrm{t}$ " del re muestreo correspondiente. Los resultados indican que en el modelo conceptual de gestión de un CTID-AS la adecuada gestión tecnológica tiene una influencia positiva en el enfoque al cliente $\left(H_{1}: \beta=0.818, p<0.001\right)$. Se comprueba igualmente que este enfoque al cliente basado en sus demandas y expectativas, tiene una influencia positiva en la administración del proyecto que se ejecutará $\left(\mathrm{H}_{2}: \beta=0.502 ; p<0.001\right)$ y en los procesos de negocios $\left(\mathrm{H}_{3}: \beta=0.646, p<0.001\right)$. Finalmente, el adecuado proceso de negocio tiene una influencia positiva en la administración del proyecto $\left(\mathrm{H}_{4}: \beta=0.414, p<0.01\right)$.

De esta manera, los valores que comprueban el ajuste del modelo son: la prueba de Residuos Medios Estandarizados RME (Standardized Root Mean Square Residual, SRMSR) que mide la diferencia entre la matriz de correlación observada y la matriz de correlaciones implícita del modelo. Un buen ajuste del 
modelo no debe exceder de 0.08 (Hu y Bentler, 1995), en este caso el ajuste se produce con valores de 0.09. Otro valor medido es el Índice de Ajuste Normado IAN (Normed Fit Index, NFI) que Bentler y Bonnet (1980) recomiendan esté por encima de 0.9; en este caso el valor alcanzado es de 0.717 que es bajo, pero Ullman (2001) advierte que esto se genera cuando las muestras son pequeñas y que, por ello, a menudo se subestima el ajuste.

\section{DISCUSIÓN FINAL}

Los resultados obtenidos del modelado de ecuaciones estructurales mediante mínimos cuadrados parciales MCP son una comprobación de la validez del modelo de gestión innovadora de un Centro de Investigación y Desarrollo auto-sostenible financieramente, que se estructura con cuatro variables latentes y 18 observables.

El modelo de gestión innovadora validado contribuye a mejorar la falta de definición de un modelo de gestión para los CTID mexicanos que les permita operar sin recursos del presupuesto gubernamental. Sus características fundamentales residen en un enfoque de sus actividades principales orientado a las demandas y expectativas del mercado, a fin de mejorar el impacto en la industria. Explica que, si los centros de investigación mexicanos que no tienen apoyos presupuestales de la federación o aspiran a no tenerlos, están en capacidad de asociar sus procesos de gestión tecnológica con las demandas y expectativas del cliente, desarrollan un favorable proceso de negocio con el cliente y luego administran eficazmente los proyectos que han sido acordados, se facilitará alcanzar la auto-sostenibilidad financiera, si se trabaja de manera sinérgica.

En la Fig. 2 se muestra un proceder metodológico contentivo de 8 pasos y la descripción de la solución para comprobar la validez del modelo de gestión innovadora de los CTID centrado en los esfuerzos en gestionar estratégicamente sus competencias clave. La aplicación de la técnica de MCP confirma mediante procedimientos robustos los modelos de gestión que no dejan lugar a dudas acerca de su fiabilidad y validez, dado el valor de la significación estadística y los coeficientes de trayectoria. Como perspectiva de la investigación el modelo de gestión innovadora, además de la validez estadística, se comprueba mediante la evidencia documental de las competencias clave y la implantación del mismo en un caso de estudio exitoso, con su efecto en el logro de la auto-sostenibilidad financiera, con base en la generación de utilidades durante un período mínimo de cinco años, lo cual inclusive sobrepasa el objetivo del presente artículo.

\section{CONCLUSIONES}

De acuerdo al trabajo presentado y a los resultados obtenidos, se pueden plantear las siguientes conclusiones principales:

1) La investigación presentada aporta al conocimiento las competencias clave necesarias y los factores determinantes para el éxito y competitividad de los CTID-AS mexicanos, así como un modelo de gestión innovadora que es validado teóricamente, incluyendo un estudio de caso exitoso, con el que el modelo propuesto se valida en la práctica.

2) El Modelo de Gestión Innovadora, fue validado con un estudio de caso exitoso de un CTID-AS mexicano, cuyo modelo de gestión innovadora comprueba el modelo teórico probado estadísticamente. Alineado con lo anterior, este CTID-AS muestra un crecimiento consistente en su auto-sostenibilidad financiera de 2007 a 2014 y razonable en los últimos 2 años, a pesar de haber decrecido en su volumen de ventas por efectos de cambios adversos (estimados temporales) en la industria a la que atiende, logrando además diversas distinciones en su impacto social y tecnológico. Con la validación teórica del Modelo de Gestión de un CTIDAS y mediante este Estudio de Caso, se da cumplimiento al objetivo general de este proyecto de investigación: diseñar y validar un modelo de gestión innovadora para un Centro Tecnológico de Investigación y Desarrollo, que le permita ser financieramente auto-sostenible, generando valor tanto para el cliente como para la organización.

3) A partir de estos resultados del estudio, se concluyó que este Centro logró asociar sus procesos de gestión tecnológica con el enfoque al cliente, basado en sus demandas y expectativas, sus procesos de negocio y administración eficaz por proyectos con la operación financieramente auto-sostenible.

4) La implantación de un modelo de gestión innovadora en Centros Tecnológicos de Investigación y Desarrollo, sobre todo en el caso de los que no tienen apoyos presupuestales de la Federación o aspiran a no tener apoyos presupuestales para su funcionamiento, presenta una oportunidad de estudio y de generación de conocimiento, razón por la cual se planteó el presente proyecto de investigación. 
5) La investigación presentada aporta al conocimiento las competencias clave necesarias y los factores determinantes para el éxito y competitividad de los CTID-AS mexicanos. Aporta además un modelo teórico de gestión innovadora que es comprobado por expertos.

\section{REFERENCIAS}

Aguilar, J. J., y E.R. Yepes, Gestión de capacidades dinámicas e innovación: Una aproximación conceptual, Revista ciencias de la Administración, ISSN 2175-8077, $8(16), \quad 280-292 \quad$ (2006). https://periodicos.ufsc.br/index.php/adm/article/view/1723/1449, Acceso 10 de enero (2018).

Aldás-Manzano, J., Partial Least Squares Path Modelling in Marketing and Management: an annotated application, https://doi.org/10.1142/9789814407724_0003, World Scientific Books, World Scientific Publishing Co. Pte. Ltd., no. 9657, (2012).

Álvarez, M. L., Competencias centrales y ventaja competitiva: el concepto, su evolución y su aplicabilidad. Contaduría y Administración: 209, 5-22 (2003).

Bagozzi, R. P. y Y. Yi, On the evaluation of structural equation models. Journal of the Academy of Marketing Science: 16(1), 7494 (1988).

Barragán, A. Factores críticos para la gestión eficaz de proyectos de innovación en Centros Públicos de Investigación y desarrollo, Tesis de Grado de Doctor en Ingeniería, Facultad de Ingeniería, UNAM, México (2010).

Bentler, P. M.; D-G. Bonnet, Significance tests and goodness of fit in the analysis of covariance structures. Psychological Bulletin: 88(3), 588-606 (1980).

Braun M., D. Brown, G. Gunnaf, J. Leroyer, y D. Meissner. Getting more innovation from public research. Arthur D. Little, Technische Universität Dresden, EAP European School Management, (2000).

Cabanelas, J., P. Cabanelas y J. Lorenzo, J. La gestión de las relaciones con los clientes como características de la alta rentabilidad empresarial. Revista Europea de Dirección y Economía de la Empresa: 16(3) 133-148 (2007).

Casanueva, C. The acquisition of firm technological capabilities in Mexico's open economy, the case of vitro. Technological forecasting and social change: (66) 75-85 (2001).

Castellanos, O. F., Gestión tecnológica: de un enfoque tradicional a la inteligencia. Bogotá D.C., Colombia, Editorial Universidad Nacional de Colombia, Facultad de Ingeniería, Editorial Universidad Nacional de Colombia Bogotá D. C. Colombia (2007).

Castillo, C. Nuevas tendencias en la retención y mejora del talento profesional y directivo. Fundación EOI, España (2009).

Cepeda, G. y J. L. Roldan. Aplicando en la práctica la técnica PLS en la administración de empresa, Universidad de Sevilla, Facultad de Ciencias Económicas y Empresariales, Sevilla, España (2005), http://ciberconta.unizar.es/doctorado/PLSGabrielCepeda.pdf, acceso 10 de enero (2018).

Chin, W. W., B.L. Marcolin y P.R. Newsted, A partial least squares latent variable modeling approach for measuring interaction effects: Results from a Monte Carlo simulation study and an electronic-mail emotion/adoption study. Information Systems Research: 14, 189-217 (2003).

Chin, W.W. The Partial Least Squares approach to structural equation modeling. In: Marcoulides, G.A. (ed), Modern Methods for Business Research, Mahwah, NJ: Lawrence Erlbaum Associates, 295-336 (1998).

Chun, H. y S. Keles, Partial least squares regression for simultaneous dimension reduction and variable selection. DOI: 10.1111/j.1467-9868.2009.00723.x. Statistical Methodology 72 (1). 3-25 (2010).

Colunga, A. S., Molina V. M. y M. C. Armenteros, Determinant Factors for Success in Self-Sustaining Research \& Development Technology Centers, Global Journal of Business Research: 10 (4), 83-93 (2016).

Colunga, A. S., Modelo de Gestión Innovadora de un Centro Tecnológico de Investigación y Desarrollo Auto-Sostenible. Tesis de doctorado. Facultad de Contaduría y Administración, Universidad Autónoma de Coahuila, Torreón, México (2017).

Estrada, S. y J. Aguirre, Los centros públicos de investigación como una herramienta de la política de ciencia, tecnología e innovación. (2013), http://www.altec2013.org/programme_pdf/927.pdf, Acceso 10 de enero (2018).

Falk, R.F. y N.B. Miller, A Primer for Soft Modeling, Akron, Ohio: The University of Akron. (1992).

Fornell, C., y D.F. Larcker, Evaluating Structural Equation Models with Unobservable Variables and Measurement Error, Journal of Marketing Research: 18(1), 39-50 (1981).

García, M. E., O.F. Castellanos y S.E. Monroy, Implementación de sistemas de inteligencia tecnológica desde la perspectiva de la complejidad. Ingeniería e investigación: 6 (2), 108-118 (2008).

Gil-Lafuente, A. M. y C. Luis-Bassa, La innovación centrada en el cliente utilizando el modelo de interferencias en una estrategia CRM. Investigaciones Europeas: 17(2), 015-032 (2011).

Gónzalez, N., J. Abad, y J.P. Lèvy, Normalidad y otros supuestos en análisis de covarianzas en Lèvy. J. (Ed.) Modelización con estructuras de covarianzas, 31-57 Editor Netbiblo, España (2006). 
Hair, J., C. Ringle, M. Sarstedt, PLS-SEM: Indeed a Silver Bullet, Journal of Marketing Theory and Practice: 19(2), 139152 (2011).

Hair, J., T. Hult, y C. Ringle, A Primer on Partial Least Squares Structural Equation Modeling (PLS-SEM). SAGE Publications, Canadá (2013).

Henseler, J., G. Hubona, y P. A. Ray, Using PLS path modeling in new technology research: updated guidelines, Industrial Management \& Data Systems, 116 (1), 2-20 (2016).

Hu, L. T., y P. M. Bentler, P. M., Evaluating model fit. In R. H. Hoyle (Ed.), Structural equation modeling Concepts, issues, and applications, 76-99 Thousand Oaks, CA: Sage. (1995).

Hlupic, V. Knowledge and Business Process Management. Idea Group Inc. Hershey, PA, USA (2002).

Intxaurburu, G. C. y E. Velasco, El Benchmarking aplicado a la gestión de innovación. Revista de Dirección y Administración de Empresas: (17), 33-46 (2010).

Kwong, K., Partial Least Squares Structural Equation Modeling (PLS-SEM) Techniques Using SmartPLS. Marketing Bulletin 24, Technical Note 1, (2013).

Leitner, K.-H., y C. Warden, Managing and reporting knowledge-based resources and processes in research organisations: specifics, lessons learned and perspectives. Management Accounting Research: 15, 33-51 (2004).

Mandeville, P. B., ¿Por qué se deben centrar las covariables en regresión lineal? Ciencia UANL: 11(3), Julio-septiembre, 300-305 (2008).

McIntosh, C. y J. R. Edwards, Reflections on Partial Least Squares Path Modeling. Organizational Research Methods (ORM), 17(2), 210-251 (2014).

Merritt, H. La vinculación industria-centros tecnológicos de investigación y desarrollo: el caso de los centros CONACYT de México. Análisis Económico: XXII (49), Primer Cuatrimestre, 149-168 (2007).

Miranda-Zapata, E., y M. A. Ruiz, Precisión en la recuperación de parámetros, con datos ordinales, en el Análisis de Estructura de Covarianza y el Modelo de Rutas mediante Mínimos Cuadrados Parciales. http://dx.doi. org/10.11144/Javeriana.upsy14-3.prp. Universitas Psychologica, 14(3), 985-996 (2015).

Molina, V., M. et al., Evaluation Model of Competitiveness Strategies in Small and Medium Industrial Firms. International Journal of Management Sciences and Business Research, 6(8), (2017).

Nath, P. y S. Mrinalini, S. Organization of R \& D: An evaluation of Best Practices. New York: Macmillan. (2002).

Nijssen E. J. y S. P. Douglas. Consumer World-Mindedness and Attitudes Toward Product Positioning in Advertising: An Examination of Global Versus Foreign Versus Local Positioning. Journal of International Marketing. 19(3). 113-133 (2011).

Rigby, D., Management tools 2015 - An executive's guide, Boston MA: Bain \& Company, Inc. (2015).

Ringle, C.M., Wende S. y J. M. Becker. “SmartPLS3".Boemmomgstedt: SmartPLS GmbH, http://www.smartpls.com. (2015).

Rodríguez-Pinto, J., Rodríguez-Escudero A. I. y J. Gutiérrez-Cillán. Order, positioning, scope and outcomes of market entry. Industrial Marketing Management, 37, 154-166 (2008).

http://www.imasdmasmk.es/images/_115521294201247Rodr\%C3\%ADguez\%20et\%20al2008.pdf. Acceso 10 de enero (2018).

Roldán, J. L. y M. J. Sánchez-Franco Variance-Based Structural Equation Modeling: Guidelines for Using Partial Least Squares in Information Systems Research. DOI: 10.4018/978-1-4666-0179-6.ch01. Research Methodologies, Innovations and Philosophies in Software Systems Engineering and Information Systems, (2012).

Rönkkö, M., The Effects of Chance Correlations on Partial Least Squares Path Modeling. https://doi.org/10.1177/1094428114525667. Organizational Research Methods (ORM) 17(2). 164-181, (2014).

Sánchez, D. y R. Álvarez, De la planeación estratégica a la planeación tecnológica. La búsqueda de ventajas competitivas sostenibles en un ambiente global. ISSN 0121-0777, El Hombre y la máquina, 17 (24), 34-35 (2005), http://www.redalyc.org/articulo.oa?id=47812408004, Acceso 10 de enero (2018).

Ullman, J. B. Structural equation modeling In. Using Multivariate Statistics by B.Tabachnick, 653-771 Needham Heights, MA (2001). 
